\title{
Intra-operative Monocular 3D Reconstruction for Image-Guided Navigation in Active Locomotion Capsule Endoscopy
}

\author{
Gastone Ciuti*1, Marco Visentini-Scarzanella*2, \\ Alessio Dore ${ }^{3}$, Arianna Menciassi ${ }^{1}$, \\ Paolo Dario ${ }^{1}$ and Guang-Zhong Yang ${ }^{3}$ \\ ${ }^{1}$ The BioRobotics Institute, Scuola Superiore Sant'Anna, Italy \\ 2 Communications and Signal Processing Group, Imperial College London, United Kingdom \\ ${ }^{3}$ The Hamlyn Centre for Robotic Surgery, Imperial College London, United Kingdom \\ * These authors contributed equally to this work.
}

\begin{abstract}
Wireless capsule endoscopy (WCE) can be considered an example of 'disruptive technology' since it represents a bright alternative to traditional diagnostic methodologies. However, currently available clinical products are passive devices whose locomotion is driven by natural peristalsis, with the drawback of failing to capture the images of potential pathological gastrointestinal tract regions, due to the impossibility to control the camera's motion and orientation. As a consequence, many research groups are working to develop active locomotion devices and endoscopic platforms that allow WCE to be performed in a totally controlled manner.

In this paper we propose a system to reliably calibrate a Shape-from-Shading system to recover the unknown scale factor immediately prior to a WCE procedure. The system would allow to reconstruct in a metrically accurate fashion the surfaces obtained intraoperatively from the WCE capsule, which can be exploited for accurate trajectory planning and ultimately automatic WCE active capsule navigation. To the best of our knowledge, this is the first practical system for Shape-from-Shading calibration aimed at guidance applications that will finally be applied to an endoscopic active locomotion platform developed by the authors.
\end{abstract}

\section{INTRODUCTION}

Wireless Capsule Endoscopy (WCE) has the potential to dramatically reduce invasiveness and pain of traditional gastrointestinal (GI) diagnostic and surgical procedures, paving the way to mass screening of the GI tract [1]. WCE potentially enables inspection of the GI tract without patient discomfort or need for sedation, and thus obviates the risks associated with traditional endoscopy [2]. As a first-generation 'disruptive technology', WCE still presents a number of limitations, e.g., the inability to control locomotion and camera orientation. As demonstrated by recent comparative studies [3], these open issues make traditional endoscopic techniques still superior to WCE.

Different approaches have been proposed for controlled locomotion but currently the most promising solution seems to be magnetic steering, under investigation by Olympus Inc. (Tokyo, Japan) and Siemens Healthcare (Erlangen, Germany) [4] as well as by the authors in [5], [6]. While promising advances have been made to control precise capsule locomotion, progress is still necessary in terms of navigation systems to close the control loop and enable automatic or semi-automatic navigation of endoscopic capsules, with the potential for greatly reduced procedural time and greater clarity of images.

Traditional methods for vision-based 3D reconstruction employed in minimally invasive surgery require the use of stereo cameras [7]-[10] which, while present on modern stereo laparoscopes and robotic platforms, are not found in WCE systems due to physical constraints. Monocular 3D reconstruction techniques such as MonoSLAM [11], [12] rely on completely static target structures, visual features that can be efficiently tracked, and a fast frame rate generating an abundance of viewpoints that can be used for structure recovery; a set of requirements that is not suitable for a typical WCE setting. Another vision-based guidance method that has seen early applications to endoscopy and minimally invasive surgery is Shape-from-Shading (SFS) [13]-[16], where the $3 \mathrm{D}$ structure of the visualized scene is reconstructed from a single monocular camera without any further information required. While SFS algorithms namely only require the target surface to be made of a material with uniform surface reflectance properties - which is the setting of GI applications - there have been no clinical applications of the algorithms without the requirement of detailed pre-operative scan data. This is due to the fact that surfaces reconstructed with SFS systems are not localized within a metric space; rather, they are linearly scaled with an unknown factor related to the surface albedo. Because of this inability to correctly recover metric distances, vision-based systems relying exclusively on guidance from SFS modules have so far not found any practical applications.

In this paper, the authors propose a complete calibration procedure to recover the surface albedo prior to the endoscopic procedure, followed by a system for SFS-based intra-operative guidance. Given the tissue uniformity of GI environments, the recovered albedo can in turn be used for intra-operative metric 3D reconstruction from SFS, accurate trajectory planning and ultimately automatic capsule navigation. The proposed system is ideally suited to platforms with self-steering capabilities [5] but it is also applicable to general capsule platforms. More specifically, even with traditional passive locomotion capsules, the 3D surfaces obtained can still be used for accurate anatomy reconstruction and augmented reality. To the best of our knowledge, this is the 
first practical system for SFS calibration for WCE guidance purposes.

\section{BACKGROUND}

\section{A. Active locomotion capsule endoscopy}

Active locomotion is mandatory for steering and navigation control of capsule devices, in order to provided a reliable diagnosis and minor therapeutic procedures in situ. In contrast, commercially available passive capsules only allow a random visual sampling of the GI tract and no control and interventional capabilities are provided.

There are mainly two strategies for active locomotion in WCE: 1) embedding miniaturized actuators on board; 2) exploiting external actuation systems, namely magnetic fields. With regards to the first method, different mechanisms have been developed by exploiting paddles and linear actuators [17], or motorized legs [18]-[22]. Although internal locomotion would grant significant advantages, such as the potential to locally manipulate tissue away from the camera, a critical drawback is that it is power intensive, thus making long operation times unfeasible. As an alternative, external locomotion approaches based on magnetic fields currently represent the most promising solution for WCE. A magnetic steering technology for gastric examination based on electromagnets was designed by Olympus Inc. and Siemens Healthcare. The system includes an Olympus capsule endoscope and a Siemens guidance system equipped with magnetic resonance imaging and computed tomography facilities [4]. A permanent magnet approach was investigated by Given Imaging Inc. (Yoqneam, Israel): a wireless PillCam colon-based capsule endoscope was modified to include neodymium-iron-boron magnets and was manipulated through a handheld external magnet in the oesophagus and stomach [23]. Another example of robotic magnetic WCE control was proposed by Carpi et al., who used the Niobe navigation system for an accurate steering of a magnetically modified PillCam video capsule [24].

A still open issue in capsule endoscopy is the leak of a reliable localization strategy compatible with magnetic locomotion for providing position and orientation feedback for a self-autonomous steering. The use of an image-based strategy would represent a promising solution for intraoperative automated guidance.

\section{B. Shape-from-Shading $3 D$ reconstruction}

Surface reconstruction with SFS is achieved through an inversion of the image irradiance equation. Essentially, given an a priori reflectance model describing the physical formation process behind the captured image, its mathematical representation is inverted in order to recover dense surface distance and normal information.

Common to all modern practical SFS systems is a perspective camera model and a Lambertian reflectance model assumption, while the light source has been assumed to be, in turn, at infinity [14], or close to the surface either at the optical center [25] or away from the visual axis [16], [26]. For the SFS problem to be well-posed, it was proven that the light source has to be modeled close to the target surface [25], [16], [26] in order to take into account the light attenuation term proportional to the distance between the light source and the surface.

However, the reconstructed surfaces do not lie within a metric space, but they are linearly scaled by an unknown factor based on the surface reflectance properties - the surface albedo. Therefore only shape, not distance, information is available. This is the case for the systems in [14] and [25], for which no practical navigation application is possible. Other systems proposed in [15] and [16] use shape information for intra-operative navigation. However, they recover the scale through iterative closest point registration with rigid preoperative data, which would be unfeasible for the deformable GI tract environment.

Another SFS-based approach was proposed for WCE procedures [27]. However, it has to be emphasized how the proposed approach does not attempt to recover metric distance, rather it is a visualization tool to aid the diagnostic procedure. Moreover, the SFS technique adopted is based on the Tsai-Shah method which models the capture device as an orthographic camera with an infinitely distant light source, making the overall system mathematically ill-posed. This can result in an ambiguous interpretation of concave/convex surfaces and, ultimately, incorrect visualization.

A more recent SFS technique attempts to recover the surface albedo to obtain metric distance by exploiting geometric properties of surface specularities [26]. However, this requires specularities to be present in the image and relies on the presence of a method for online robust separation of specular and diffuse components, which can be difficult under highly focused illumination.

It is therefore crucial for a SFS-based navigation system without pre-operative scan data to recover the missing albedo needed for metric reconstructions. Here we propose a platform-independent practical calibration system that can be used for this purpose as a first step towards automatic WCE capsule navigation. We also show how the recovered albedo can be used to metrically scale intra-operative reconstructions robustly through a cross-validation procedure.

\section{SYSTEM WORKFLOW}

The proposed calibration system requires the camera that will be used during the WCE procedure together with a way of reliably controlling the camera position.

To demonstrate our calibration concept, we used a standard STORZ laparoscope model together with a synthetic colon model kept at a stable $2 \mathrm{mmHg}$ insufflation level by an external endoscopic insufflator (Surgiflator-40, Word of Medicine AG, Germany), but the same procedure could be implemented with similar equipment or in-vivo with relatively few modifications.

The laparoscopic camera was first calibrated to characterize its radiometric response and internal parameters. The laparoscope was then inserted in the colon for a total length of $100 \mathrm{~mm}$, with images captured at insertion intervals of $5 \mathrm{~mm}$. The complete experimental setup is shown in Fig. 1 and precise insertion steps have been addressed through 


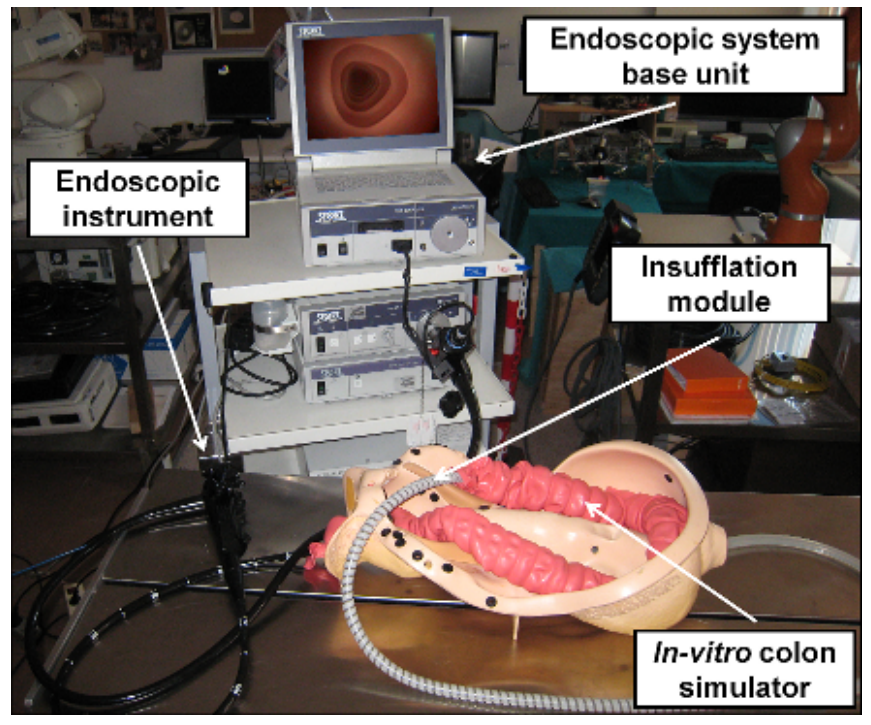

Fig. 1: Experimental in-vitro test bench for the calibration procedure.

a high resolution measuring scale fixed on the endoscope. The images were then pre-processed to remove deviations from the assumed Lambertian reflectance model and fed to our SFS algorithm for surface reconstruction. Finally, visual features were matched between consecutive frames.

To recover the unknown surface albedo, we considered the ratio between the difference of the reconstructed depth for matching features and the known $5 \mathrm{~mm}$ displacement used during calibration. A distribution of the ratios recovered for all matching features was created for each consecutive frame pair. Given uniform surface characteristics throughout the GI tract, the calculated distributions should all indicate a single scale factor that can then be calculated during calibration, stored and used for metric 3D reconstruction and capsule trajectory planning during WCE procedures in the same subject without the need for controlled movement.

The stages of the calibration algorithm will be discussed in details in the following sections and consist of camera geometric and radiometric calibration, image pre-processing, SFS 3D reconstruction, and feature matching with scale factor recovery. During intra-operative guidance using the albedo recovered during calibration, the pipeline consists of image pre-processing, SFS 3D reconstruction and scaling with the calculated albedo, and trajectory planning. Results on the effectiveness and accuracy of the proposed method will be presented in section IV

In this framework the capsule would be reliably steered in according to the calculated trajectory by means of a roboticassisted magnetic propulsion [6] and controlled exploiting a highly precise localization, such as the one proposed by the author in [28], making the strategy assumptions not so far from a real scenario.

\section{A. Camera calibration}

The endoscopic camera was calibrated prior to insertion with a standard checkerboard in order to determine its

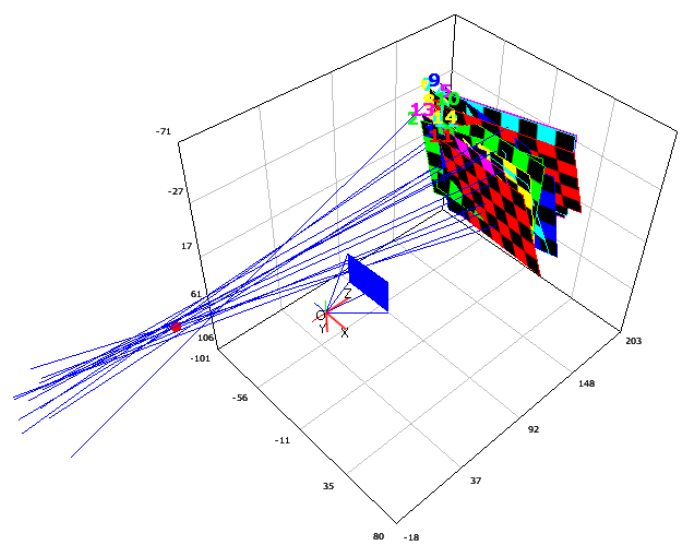

Fig. 2: Light source position calibration. The position of the light source is accurately determined with the method by [29] to better characterize the SFS setting.

intrinsic parameters. To further characterize the endoscopic camera-light source setup, a reflective plastic film was overlaid to the checkerboard in order to provide specular highlights that allowed to calculate the exact spatial location of the light source with respect to the camera according to the method presented in [29]. The measured translation of the light source with respect to the camera center was of $(x, y, z)=(3.5,2.2,5.0) \mathrm{mm}$. An example of the output from the light calibration phase is shown in Fig. 2

Given a surface reflectance model mathematically defining the image irradiance, every SFS algorithm assumes a linear relationship between this irradiance and the captured brightness. However, due to automatic camera post-processing and specific charge-coupled device element sensitivities, this is seldom the case.

Therefore, a radiometric calibration was carried out to determine the camera radiometric response function and invert it in order to linearize the relationship between incoming image irradiance and the perceived brightness output. This was done with images of a Macbeth color chart illuminated by the endoscope light source. The exposure time was fixed throughout calibration and insertion, while all automatic color balancing processes were deactivated. Vignetting was minimized by the use of a uniform ambient light together with the scope light source. Among all families of functions tried, gamma functions were found to be the best fitting in the Least-Square sense. The functions for the three color channels were then inverted to determine the radiometric response. Given this information, it is then possible to linearize the brightness of incoming frames on-the-fly. Exponential functions describing per-channel camera radiometric response are $1.6 x^{0.8758}, 29.16 x^{0.3615}$ and $16.52 x^{0.4424}$, respectively for the red, green and blue channel.

\section{B. Image processing}

Endoscopic images can present specular highlights, defined as bright spots of light appearing on shiny object when illuminated. This is due to the highly focused bright lighting interacting with water and mucosal tissue. Even 
though highlights are usually important in computer vision as they provide a strong visual cue for the shape of an object and its location with respect to light source in the scene, they represent a critical problem for the resolution of the SFS algorithm because of their deviation from the assumed Lambertian reflectance.

Therefore, it is important to remove specular highlights prior to SFS reconstruction. Specular highlights are detected in the original frame through thresholding areas of high intensity and low saturation. The areas delimiting the detected regions were then dilated in order to fill any local holes in the detection and used as selection masks for an image inpainting process aimed at populating the specular areas with intensity values from neighboring pixels. A Gaussian filter was then applied to the frame for an overall smooth result without edge artifacts between the inpainted areas and their surroundings. Finally, the frame was reconstructed by combining pixels from the original (Fig. 3a) and the inpainted and filtered frames, respectively for the area outside and inside the inpainting mask. Median filtering was then applied on the resulting frame to remove salt-and-pepper noise (Fig. 3b).

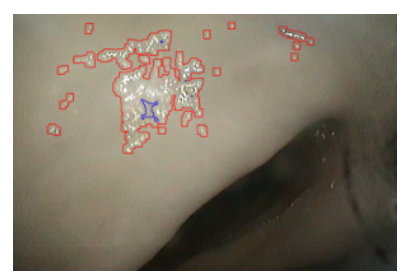

(a)

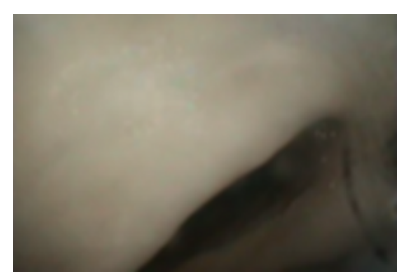

(b)
Fig. 3: Specular highlight removal. (a) Original frame with selected specular highlight areas. (b) Final frame after specular highlight removal.

\section{Shape-from-Shading 3D Reconstruction}

To reconstruct the $3 \mathrm{D}$ structure of the visualized endoscopic environment, a SFS technique is used. In its general formulation for Lambertian surfaces, SFS can be seen as an inverse problem attempting to reconstruct the scene shape from a single monocular frame by solving the irradiance equation:

$$
I=\rho \frac{1}{r^{2}} \frac{\mathbf{n} \cdot \mathbf{l}}{\|\mathbf{n}\|\|\mathbf{I}\|}
$$

In equation 1, the SFS problem is expressed as recovering information about the surface normal $\mathbf{n}$ based on the knowledge of the light source position $\mathbf{l}$, the surface albedo $\rho$ to be recovered, and the perceived image intensity $I$ for all pixels. Despite it being an underconstrained problem, wellposedness has been shown under configurations where the light source is close to the target surface [26] and the distance attenuation term $\frac{1}{r^{2}}$ between the light source and the target surface is not ignored.

In our configuration, we adopt the system proposed by Visentini-Scarzanella et al. [26], where a single light source
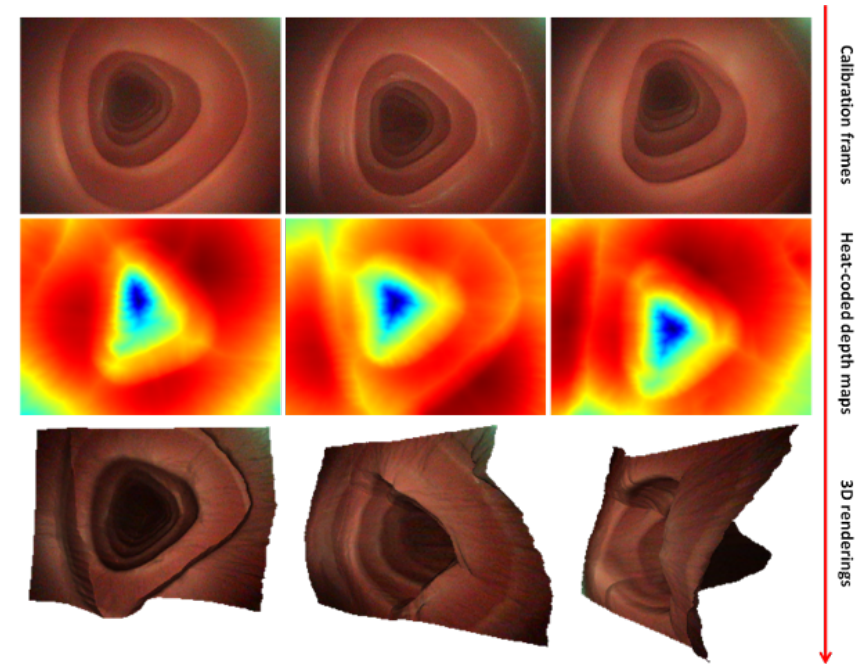

Fig. 4: Selected calibration frames (top row), their heat-coded depth maps (middle row) and projected renderings (bottom row).

away from the optical center is considered instead of two like in the original formulation. The resulting SFS partial differential equations is characterized with the information from the camera and light calibration procedure and then solved through a Lax-Friedrichs sweeping scheme. The code for the SFS stage of the algorithm has been made publicly available on the author's page ${ }^{1}$ Contrarily to the formulation in [26] where the recovery of the albedo relies on robust separation of specular and diffuse components which might be difficult in practice, with our additional calibration procedure it is possible to recover this scale factor prior to the procedure and dynamically apply it for in-vivo monocular metric structure recovery.

In Fig. 4, selected endoscopic frames are shown together with the depth maps obtained from the SFS algorithm. The calculated depth was then projected using the calibrated camera information to obtain realistic $3 \mathrm{D}$ renderings that can be used for trajectory planning or augmented reality.

\section{Feature matching and scale factor recovery}

In this step, pairs of consecutive frames (separated by a distance of $5 \mathrm{~mm}$ ) were processed with a feature detection and matching algorithm in order to measure the SFS-obtained depths of matching pixels in the two frames. On each pair, a Shi and Tomasi detection phase was followed by a Pyramidal Lucas-Kanade feature matching phase [30] were executed. Since only a relatively small number of feature matches are required for the albedo calculation, the Lucas-Kanade tracker was chosen because of its speed and ease of implementation. A typical screenshot of the detection and matching algorithm is reported in Fig. 5 .

Each frame pair was processed in the same manner and for each pair of matched pixels an inferred albedo was obtained, defined as the ratio between the difference in SFS-depth and

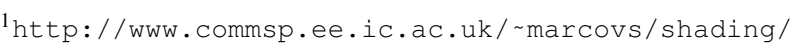




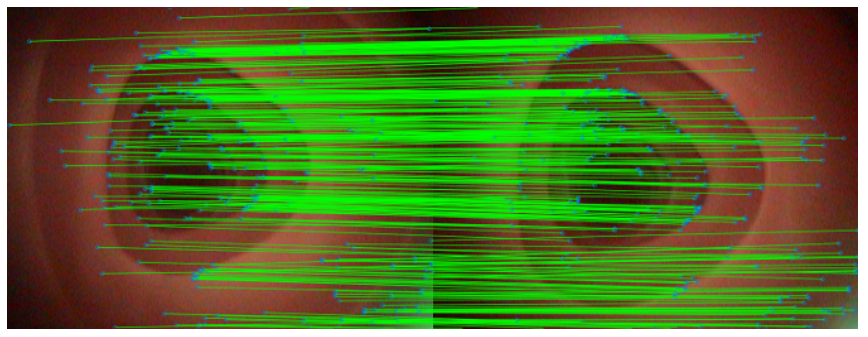

Fig. 5: Feature matching stage with the Lucas-Kanade tracker. Reconstructed depth of matched features between consecutive frames will be used to determine the SFS scaling factor

the known distance of $5 \mathrm{~mm}$. Finally, the overall mean and variance of the albedo for all the image pairs was calculated as presented in section IV] This matching step is only carried out during the calibration procedure, since it is used to calculate the albedo that can then be used directly for metric scaling.

\section{E. Mesh skeletonisation}

For WCE capsule navigation, the optimal navigation path can be directly computed as the centerline of the 3D model, reconstructed with the SFS algorithm and scaled back to metric dimensions with the calculated albedo. This path is optimal in the sense that it allows the capsule to be always in the center of the lumen avoiding occlusions and providing the most complete view of the GI tract. The mesh of the 3D model is first voxelised to obtain a full volume through the methodologies presented in [31], [32]. This procedure allows the transformation of the polygonal representation into a volumetric description by a parity count approach. Parallel rays are cast through the model and the voxels are considered as internal to the mesh if the ray intersects an odd number of times the mesh polygons. Then, the centerline (or skeleton) of the volume is obtained with the thinning algorithm presented in [33]. In particular, the volume is reduced to lines with a single voxel thickness by an iterative erosion procedure.

\section{VALIDATION AND RESULTS}

An experimental validation was performed in a plastic colon simulator to verify the calibration procedure allowing to recover the surface albedo for the SFS algorithm. A sequence of 20 images was taken at $5 \mathrm{~mm}$ intervals while moving the instrument forward up to $100 \mathrm{~mm}$ from insertion. The camera orientation was maintained stable throughout the procedure. Each consecutive image pair was processed and the albedo was calculated as the ratio between the difference in depth for each matched pixel and the known $5 \mathrm{~mm}$ step. On average, 233 features were matched per frame pair. While such an exiguous number of available features would be too low for other reconstruction algorithms as it accounts for $0.05 \%$ of the image pixels, it is sufficient to populate probability distributions to infer the unknown albedo. In particular, a distributions analysis per frame-pair was followed by a leave-one-out cross validation, as reported in the next subparagraphs.

\section{A. Distributions analysis per frame-pair}

Data relative to the extracted scale factors for all matched pixels for the each image pair was fitted to a Weibull continuous probability distribution as it exhibited strong nonGaussian properties that best matched the Weibull characteristics [34]. Means and variance parameters were extracted from the fitted probability density functions for each image pair.

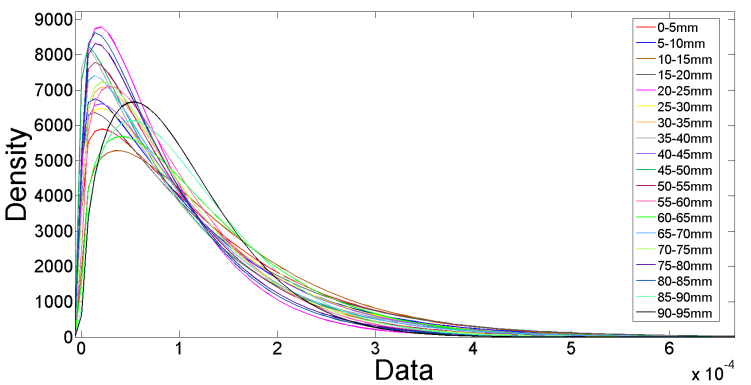

Fig. 6: Weibull continuous probability distribution of the scale factors for each consecutive frame pair.

As shown in Fig. 6, the 19 distributions all have a similar trend and Weibull parameters. This indicates the consistency and repeatability of the SFS algorithm in environments with uniform surface characteristics. Finally, the overall mean and variance of the scale factor for all the image pairs was calculated by fitting the data relative to each distribution's mean to a Gaussian distribution, visualized in Fig. 7] Numerically, the distribution of the reconstruction's scale factor is centered at $1.06 \cdot 10^{-4}$, with a variance of $1.91 \cdot 10^{-10}$.

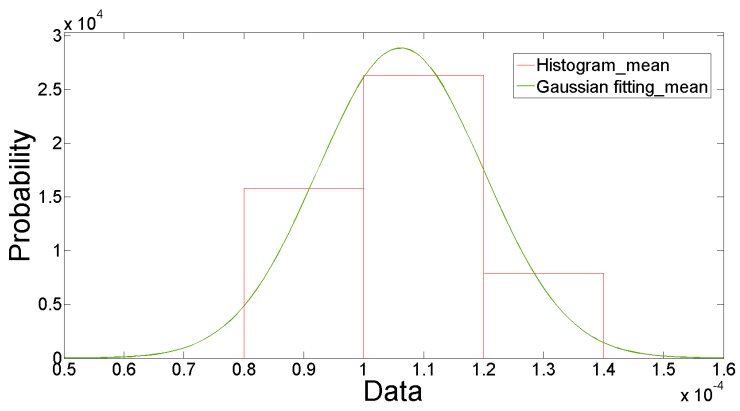

Fig. 7: Overall distribution of the mean scale factor for each consecutive image pair.

\section{B. Leave-one-out cross-validation}

In order to validate the metrically scaled reconstructions from the SFS algorithm using the calculated albedo, a leave-one-out cross validation test was performed on the extracted frames. For each iteration, the albedo was therefore calculated as the mean scale for all image pairs except for the one used for the validation. 
Then, for each image pair considered in the crossvalidation, the raw difference in depth from the SFS algorithm between matching features was divided by the albedo obtained by the remaining image pairs. The distribution of the recovered depths after scaling for each pair in the crossvalidation is shown in Fig. 8 .

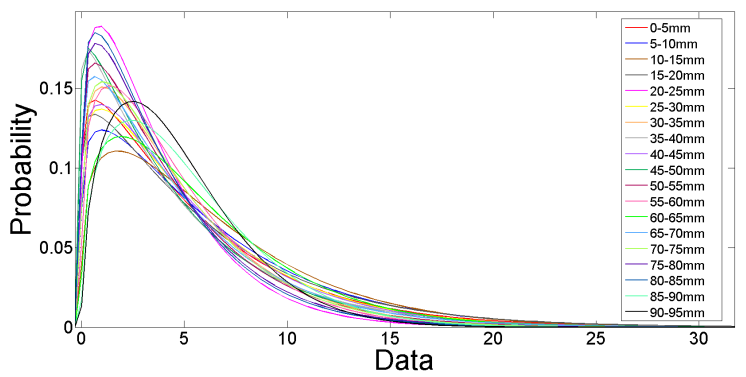

Fig. 8: Leave-one-out cross-validation: Weibull distribution of the recovered step size for all matched features in each consecutive frame pairs.

Finally, validation was achieved through plotting the mean recovered step size for each iteration of the leave-one-out process. The means neatly fit a Gaussian distribution centered at $5.0047 \mathrm{~mm}$ with a variance of 0.4733 against a groundtruth step size of $5.00 \mathrm{~mm}$. The distribution of the recovered mean step sizes is shown in Fig. 9 Table I numerically presents the mean recovered step size for each iteration of the process.

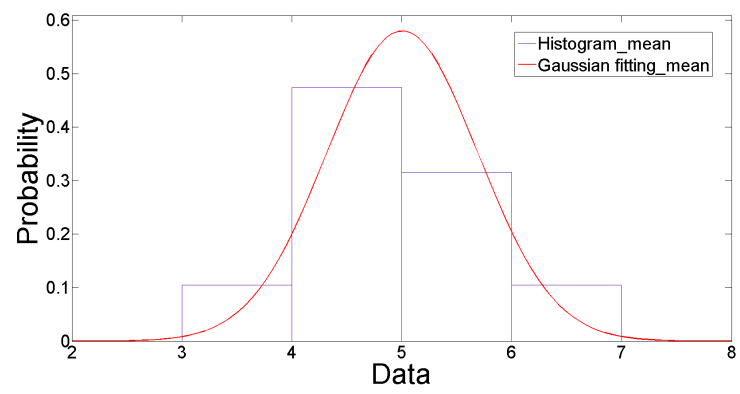

Fig. 9: Leave-one-out cross-validation - Gaussian distribution of recovered mean step size for each frame pair.

The recovered scale factors were used to scale up the reconstructed meshes and planned trajectories to meaningful metric distances. A visualization of the planned capsule trajectory is shown in Fig. 10

The rendering shows a cone-like shape of the reconstructed volume, which is unrealistic given the cylindrical shape of the colon. This result is due to the limited camera resolution combined with the high perspective effect of the input images, yielding a very limited number of distant voxels connected to the rest of the volume.

In a practical system this effect would be inconsequential to the guidance since trajectory can be recalculated at every acquired frame, hence well before the capsule would reach

\begin{tabular}{ccccc} 
& $\mathbf{0 - 5} \mathbf{~ m m}$ & $\mathbf{5 - 1 0} \mathbf{~ m m}$ & $\mathbf{1 0 - 1 5} \mathbf{~ m m}$ & $\mathbf{1 5 - 2 0} \mathbf{~ m m}$ \\
\hline MEAN & 6.00868 & 5.49713 & 6.27875 & 5.9544 \\
VARIANCE & 27.9874 & 25.2638 & 26.1658 & 30.2054 \\
\hline & $\mathbf{2 0 - 2 5} \mathbf{~ m m}$ & $\mathbf{2 5 - 3 0} \mathbf{~ m m}$ & $\mathbf{3 0 - 3 5} \mathbf{~ m m}$ & $\mathbf{3 5 - 4 0} \mathbf{~ m m}$ \\
\hline MEAN & 3.7724 & 5.39844 & 4.7507 & 4.99337 \\
VARIANCE & 10.1702 & 22.3837 & 16.2631 & 23.1794 \\
\hline & $\mathbf{4 0 - 4 5} \mathbf{~ m m}$ & $\mathbf{4 5 - 5 0} \mathbf{~ m m}$ & $\mathbf{5 0 - 5 5} \mathbf{m m}$ & $\mathbf{5 5 - 6 0} \mathbf{~ m m}$ \\
\hline MEAN & 5.282 & 4.82001 & 4.57023 & 4.54171 \\
VARIANCE & 21.3927 & 21.0144 & 16.695 & 13.2707 \\
\hline & $\mathbf{6 0 - 6 5} \mathbf{~ m m}$ & $\mathbf{6 5 - 7 0} \mathbf{~ m m}$ & $\mathbf{7 0 - 7 5} \mathbf{~ m m}$ & $\mathbf{7 5 - 8 0} \mathbf{~ m m}$ \\
\hline MEAN & 5.68684 & 4.89343 & 4.62578 & 4.13766 \\
VARIANCE & 19.7683 & 19.5627 & 15.2519 & 13.0724 \\
\hline & $\mathbf{8 0 - 8 5} \mathbf{~ m m}$ & $\mathbf{8 5 - 9 0 m m}$ & $\mathbf{9 0 - 9 5} \mathbf{~ m m}$ & OVERALL \\
\hline MEAN & 3.9924 & 5.15181 & 4.73379 & $\mathbf{5 . 0 0 4 7 1 2 1 1}$ \\
VARIANCE & 12.1925 & 13.5302 & 10.4494 & $\mathbf{0 . 4 7 3 3 7 7}$
\end{tabular}

TABLE I: Mean and variance of recovered step size for each iteration of the validation process

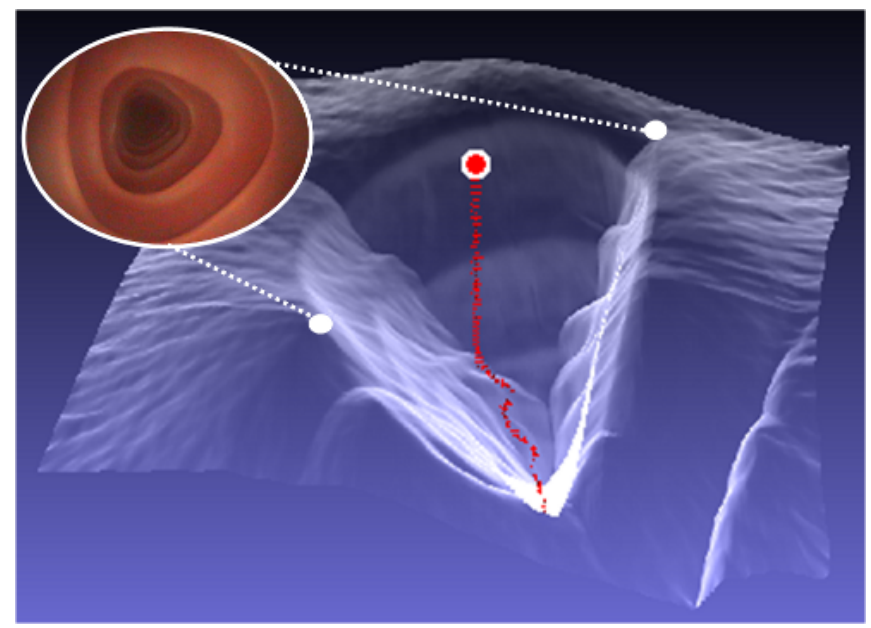

Fig. 10: Planned capsule trajectory (red) going through a reconstructed section of the synthetic colon model, rendered semi-transparently for clarity. The capsule location is indicated by the red and white dot.

the far end of the visualized volume where the visibility and morphological accuracy are limited.

\section{CONCLUSIONS AND FUTURE WORK}

In this paper we propose a system to reliably calibrate a SFS system in order to enable intraoperative metrically accurate $3 \mathrm{D}$ reconstruction during a robotic-aided active locomotion WCE procedure. The reconstructed surfaces can be exploited for augmented reality, accurate trajectory planning and ultimately to close the control loop and enable automatic WCE active capsule navigation. A mean recovered distance of $5.0047 \mathrm{~mm}$ with a variance of 0.4733 against a groundtruth step size of $5.00 \mathrm{~mm}$ obtained with the leave-one-out cross-validation demonstrated the efficiency and reliability of the proposed system as a first and fundamental step towards automatic WCE navigation.

To the best of our knowledge, this is the first practical application of SFS for guidance purposes without any neces- 
sary knowledge of pre-operative data. In a real scenario the endoscopic device will be accurately steered in according to the calculated trajectory by means of a robotic-assisted magnetic propulsion [5], [6], closing the capsule control motion loop throughout a highly precise localization strategy, such as the one proposed by the author in [28].

Notwithstanding, together with this novelty, our contributions lie in the development of a completely platform independent system that can be applied to any capsule. For active locomotion devices, our described trajectory planning procedure can be adopted as a first step towards automatic steering. For passive devices, the reconstructed environments can be used to enhance visualization, reconstruct the environment and aid diagnosis.

Future work will be dedicated to the integration of the implemented reconstruction algorithm into the robotic arm control system, previously developed by the authors [5]. The final aim is to develop a complete automated active locomotion WCE platform. In order to reliably match the magnetically dragged capsule motion on the defined ideal trajectory, sensors will be integrated on-board to provide a closed-loop feedback, thus enabling an accurate step size motion control and a greater calibration accuracy.

\section{ACKNOWLEDGEMENTS}

The authors wish to thank A. Verbeni, V. Castelli, M. Nardi and D. Stoyanov for their help in the setup development and in performing the experimental test.

\section{REFERENCES}

[1] Salmore R. Our heritage: a history of gastroenterology and gastroenterology nursing. Gastroenterol Nurs. 1998;21(2):40-43.

[2] Reavis K, Melvin W. Advanced endoscopic technologies. Surg Endosc. 2008;22:1533-1546.

[3] Moglia A, Menciassi A, Dario P, Cuschieri A. A clinical update: endoscopy for small bowel tumours. Lancet. 2007;370:114-116.

[4] Rey JF, Ogata H, Hosoe N, Ohtsuka K, Ogata N, Ikeda K, et al. Feasibility of stomach exploration with a guided capsule endoscope. Endoscopy. 2010;42(7):541-545.

[5] Ciuti G, Valdastri P, Menciassi A, Dario P. Robotic magnetic steering and locomotion of capsule endoscope for diagnostic and surgical endoluminal procedures. Robotica. 2010;28(2):199-207.

[6] Ciuti G, Donlin, R Valdastri P, Arezzo A, Menciassi A, Morino M, et al. Robotic versus manual control in magnetic steering of an endoscopic capsule. Endoscopy. 2010;42:148-152.

[7] Stoyanov D, Visentini-Scarzanella M, Pratt P, Yang GZ. Real-Time Stereo Reconstruction in Robotically Assisted Minimally Invasive Surgery. In: MICCAI; 2010. p. 275-282.

[8] Pratt P, Stoyanov D, Visentini-Scarzanella M, Yang GZ. Dynamic Guidance for Robotic Surgery Using Image-Constrained Biomechanical Models. In: MICCAI; 2010. p. 77-85.

[9] Visentini-Scarzanella M, Mylonas GP, Stoyanov D, Yang GZ. BRUSH: A Gaze-Contingent Virtual Paintbrush for Dense 3D Reconstruction in Robotic Assisted Surgery. In: MICCAI; 2009. p. 353-360.

[10] Mountney P, Yang GZ. Motion Compensated SLAM for Image Guided Surgery. In: MICCAI; 2010. p. 496-504.

[11] Davison AJ. Real-Time Simultaneous Localisation and Mapping with a Single Camera. In: ICCV; 2003. p. 1403-1410.

[12] Newcombe RA, Davison AJ. Live dense reconstruction with a single moving camera. In: CVPR; 2010. p. 1498-1505.

[13] Okatani T, Deguchi K. Shape Reconstruction from an Endoscope Image by Shape from Shading Technique for a Point Light Source at the Projection Center. Computer Vision and Image Understanding. 1997;66(2):119-131.
[14] Tankus A, Sochen N, Yeshurun Y. Reconstruction of Medical Images by Perspective Shape-from-Shading. In: 17th International Conference on Pattern Recognition (ICPR'04). Cambridge, UK; 2004. p. 778-781.

[15] Lamata P, Morvan T, Reimers M, Samset E, Declerck J. Addressing Shading-Based Laparoscopic Registration. In: Dassel O, Schlegel WC, Magjarevic R, editors. World Congress on Medical Physics and Biomedical Engineering, September 7 - 12, 2009, Munich, Germany. vol. 25-6 of IFMBE Proceedings. Springer Berlin Heidelberg; 2009. p. $189-192$.

[16] Wu C, Narasimhan SG, Jaramaz B. A Multi-Image Shape-fromShading Framework for Near-Lighting Perspective Endoscopes. INT J COMPUT VISION. 2010;86(2-3):211-228.

[17] Kim HM, Yang S, Kim J, Park S, Cho JH, Park JY, et al. Active locomotion of a paddling-based capsule endoscope in an in vitro and in vivo experiment (with videos). Gastrointest Endosc. 2010;72(2):381387.

[18] Gorini S, Quirini M, Menciassi A, Pernorio G, Stefanini C, Dario P. A Novel SMA-Based Actuator for a Legged Endoscopic Capsule. In: Biomedical Robotics and Biomechatronics, 2006. BioRob 2006; 2006. p. 443-449.

[19] Quirini M, Menciassi A, Scapellato S, Stefanini C, Dario P. Design and Fabrication of a Motor Legged Capsule for the Active Exploration of the Gastrointestinal Tract. IEEE-ASME T MECH. 2008 April;13(2):169-179.

[20] Quirini M, Menciassi A, Scapellato S, Dario P, Rieber F, Ho CN, et al. Feasibility proof of a legged locomotion capsule for the GI tract. Gastrointest Endosc. 2008;67(7):1153-1158.

[21] Valdastri P, Webster RJ, Quaglia C, Quirini M, Menciassi A, Dario P. A New Mechanism for Mesoscale Legged Locomotion in Compliant Tubular Environments. IEEE T ROBOTIC AUTOM. 2009 Oct;25(5):1047-1057.

[22] Quaglia C, Buselli E, Webster RJ, Valdastri P, Menciassi A, Dario P. An endoscopic capsule robot: a meso-scale engineering case study. Journal of Micromechanics and Microengineering. 2009;19(10):105007.

[23] Swain P, Mosse CA, Volke F, Gerber J, Keller J. 511i: In Vivo Studies of the Potential and Limitations of Remote Control of Functional Wireless Capsule Endoscopes With Rare Earth Magnetic Inclusions in an Extra-Corporeal Magnetic Field. Gastrointest Endosc. 2010;71(5):AB123.

[24] Carpi F, Kastelein N, Talcott M, Pappone C. Magnetically Controllable Gastrointestinal Steering of Video Capsules. IEEE T BIO-MED ENG. $2011 \mathrm{Feb} ; 58(2): 231-234$.

[25] Wang GH, Han JQ, Zhang XM. Three-dimensional reconstruction of endoscope images by a fast shape from shading method. MEAS SCI TECHNOL. 2009;20(12):125801-125810.

[26] Visentini-Scarzanella M, Stoyanov D, Yang GZ. Metric depth recovery from monocular images using Shape-from-Shading and specularities. In: Proceedings of the 2012 IEEE International Conference on Image Processing (ICIP 2012); 2012. To appear.

[27] Karargyris A, Bourbakis N. Three-Dimensional Reconstruction of the Digestive Wall in Capsule Endoscopy Videos Using Elastic Video Interpolation. IEEE T MED IMAGING. 2011 April;30(4):957 -971.

[28] Salerno M, Ciuti G, Lucarini G, Rizzo R, Valdastri P, Menciassi A, et al. A discrete-time localization method for capsule endoscopy based on on-board magnetic sensing. TRANS ASME, J APPL MECH. 2012;23(1):1-10.

[29] Stoyanov D, Elson DS, Yang GZ. Illumination position estimation for $3 \mathrm{D}$ soft-tissue reconstruction in robotic minimally invasive surgery. In: IROS; 2009. p. 2628-2633.

[30] Bouguet JY. Pyramidal Implementation of the Lucas Kanade Feature Tracker Description of the algorithm. Intel Corporation Microprocessor Research Labs; 2000.

[31] Nooruddin FS, Turk G. Simplification and Repair of Polygonal Models Using Volumetric Techniques. IEEE Trans Vis Comput Graphics. 2003;9:191-205.

[32] Min P. Binvox; 2011. http://www.cs.princeton.edu/ min/binvox/.

[33] Palágyi K, Kuba A. Directional 3D Thinning Using 8 Subiterations. In: Proceedings of the 8th International Conference on Discrete Geometry for Computer Imagery, DCGI 1999; 1999. p. 325-336.

[34] Weibull W. A statistical distribution function of wide applicability. TRANS ASME, J APPL MECH. 1951;18(3):293-297. 